\title{
Focused Ion Beam Milling of Eukaryotic Cells for Determining Membrane Interaction
}

Lester Lampert, Andrew Barnum, and Jun Jiao

Portland State University, Department of Physics, Portland OR, USA

To further the development of intracellular recording devices, it is essential to determine the viability and interaction of the cell membrane with the functional portions of any proposed device. One method for directly viewing the interaction of a cell with a particular substrate and/or material is by sectioning the sample via focused ion beam (FIB) milling. Through this process, one is able to selectively probe different regions of the cell, create lamellae for use with transmission electron microscopy (TEM), and potentially create a three-dimensional reconstruction of the internal cellular structures through cryoelectron tomography [1]. Here, we report FIB milling of dendritic cells (DC 2.4) cultured onto a substrate of vertically aligned carbon nanotubes (VACNTs). Carbon nanotubes (CNTs) are a commonly chosen material for presenting various molecules into the cytosol of eukaryotic cells, whether it is used for intracellular recording or as a vector for delivering DNA, antigen, or other substances [2].

FIB sectioning and subsequent thinning of lamellae were performed with an FEI Strata 237 FIB equipped with an Omniprobe Autoprobe. VACNT substrates were grown by DC plasma enhanced chemical vapor deposition (PECVD). $3.5 \mathrm{~nm}$ of Ni was deposited onto Si substrates with $100 \mathrm{~nm}$ of thermal oxide $\left(\mathrm{SiO}_{2}\right)$ via DC sputter coating. The samples were ramped to $800{ }^{\circ} \mathrm{C}$ under $\mathrm{NH}_{3}$ followed by the introduction of $\mathrm{C}_{2} \mathrm{H}_{2}$ for a growth time of $30 \mathrm{~min}$. DC 2.4 cells were cultured in Dulbecco's Modified Eagle Medium (DMEM) with 10\% fetal bovine serum (FBS) on top of the VACNTs until they reached a confluence of $\sim 80 \%$. Shortly afterwards, the DC 2.4 samples were stained using a modified version of a tannin-osmium-thiocarbohydrazide-osmium procedure developed by Murakami et al. [3]. They were subsequently dehydrated in gradually increasing concentrations of ethanol in $\mathrm{DI} \mathrm{H}_{2} \mathrm{O}$ up to $100 \%$ and then supercritically dried. Samples were stored in a low-moisture environment prior to FIB milling.

Our results reveal that DC 2.4 cells formed networks by elongating their membranes and establishing firm contact with the VACNTs as shown in Figure 1a. Also, due to surface tension effects, once the VACNTs are dried during the preparation of the samples for SEM, FIB, and TEM, they formed bundles. As demonstrated in Figure 1d and 1e, the CNTs were engulfed by the cell membrane and gained access to the cytosol. However, the membrane does not appear to be closed, which may cause issues for the cultured cells because they will be unable to regulate intracellular entry. The CNTs are confirmed entering the cells by an EDS line scan of the Ni catalyst-filled tips as shown in Figure 1b. A lamella inspected by TEM demonstrates the entry points for CNTs as can be seen in Figure 2b. The CNTs attached to the lamella detached once lift-out was initiated.

References:

[1] A. Rigort et al, PNAS 109 (2012), p. 4449.

[2] F. J. Lawson et al, Nano Letters 13 (2012), p. 1.

[3] T. Murakami et al, Scanning Electron Microscopy 1 (1983), p. 235.

[4] The authors would like to acknowledge CEMN for their support in FIB, SEM, and TEM work. 


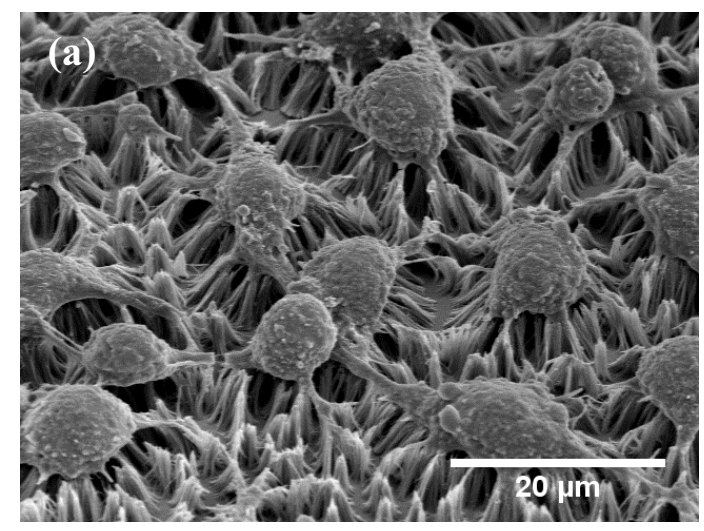

(b)
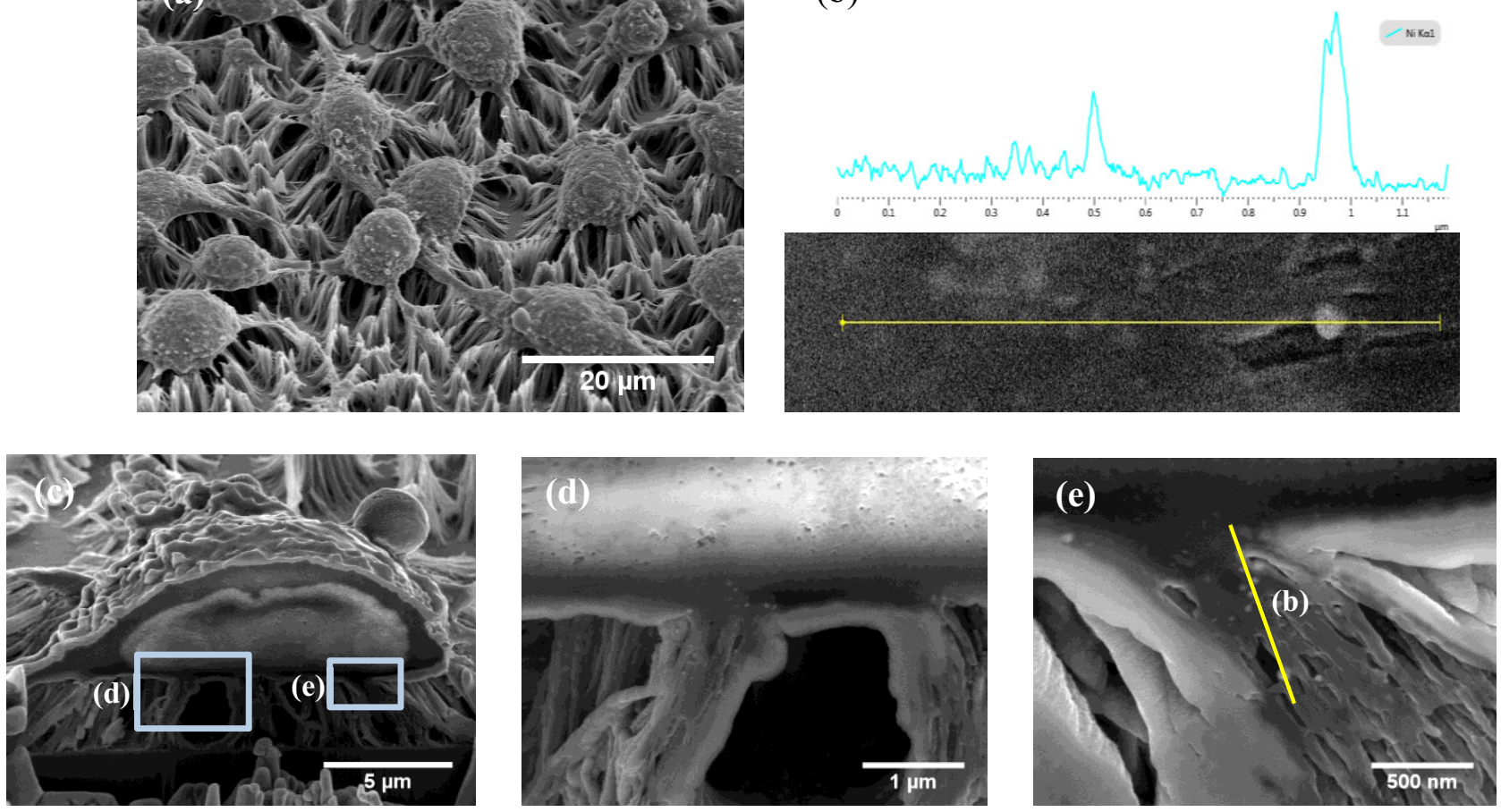

Figure 1. (a) Low-magnification of DC 2.4 cells attached to VACNTs, (b) EDS line scan across CNTs and Ni catalyst-filled tips (c) FIB cross-section, (d) CNTs seen accessing the cytosol of the cell, and (e) the cell membrane attempts to engulf the CNT bundle.
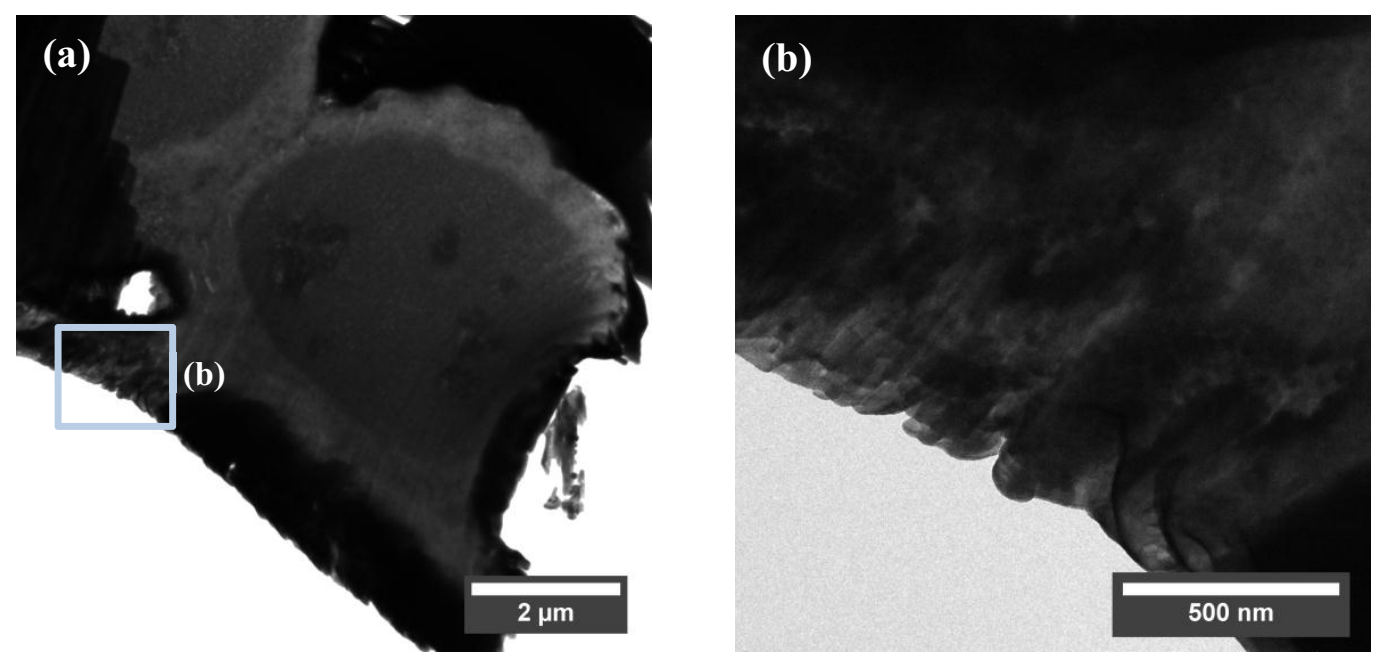

Figure 2. (a) Lamella milled by FIB showing two cells (b) Section of cell lamella where CNTs had access to the cytosol of the DC 2.4 cells. 